\section{PTH-048 ACTIVE ULCERATIVE COLITIS IS ASSOCIATED WITH ALTERED FATTY ACID PRECURSOR AVAILABILITY FOR THE EICOSANOID SYNTHETIC PATHWAY}

doi:10.1136/gut.2011.239301.449

D S Pearl,1,* D Gullick, ${ }^{2}$ J Bruemmer, ${ }^{3}$ J Brown, ${ }^{2}$ J K Shute, ${ }^{2}$ G Mills, ${ }^{2}$ T M Trebble ${ }^{1}$ Department of Gastroenterology, Portsmouth Hospitals NHS Trust, Portsmouth, UK; ${ }^{2}$ Institute of Biomedicine and Biomolecular Sciences, University of Portsmouth, Portsmouth, UK; ${ }^{3}$ Department of Environmental Sciences, University of Portsmouth, Portsmouth, UK

Introduction The polyunsaturated fatty acids (PUFA) arachidonic acid (AA), eicosapentaenoic acid (EPA), docosapentaenoic acid (DPA) and docosahexaenoic acid (DHA) are generated from dietary essential fatty acids linoleic acid (LA) and $\alpha$-linolenic acid ( $\alpha$ LNA) and direct dietary intake. AA and EPA are substrates for eicosanoid inflammatory mediators that may have critical roles in immunomodulation in Ulcerative Colitis (UC). Dietary supplementation studies alter the availability of specific PUFA (eg, EPA), but clinical studies have been disappointing leading to the hypothesis that the PUFA biosynthetic pathway in inflamed tissue may be altered. This study evaluated PUFA bioavailability in inflamed and uninflamed mucosa from UC patients and compared to matched controls.

Methods Ethical approval was obtained. Patients were prospectively recruited from outpatients' clinics. Mucosal biopsies at flexible sigmoidoscopy (FS) were taken from active UC patients within inflamed (active) and endoscopically normal proximal mucosa (internal control). Age-sex matched patients undergoing FS for IBS or similar presentations were compared (external controls). Mucosal samples were saponified, deuterated internal standards added, lipids hydrolysed and fatty acids derivatised prior to liquid-liquid extraction into heptane. PUFA were quantified by gas chromatography mass spectrometry. Data was expressed as percentage mass abundance and compared using Wilcoxon signed rank pair analysis for non-parametric data.

Results 69 active UC patients (54 paired normal/inflamed mucosa) and 69 controls were compared. No biologically significant differences were noted between endoscopically normal mucosa from UC patients and external controls other than DPA $(p<0.0025)$. Inflamed mucosa compared to both control groups demonstrated highly significant reductions in the essential fatty acids LA and $\alpha$ LNA $(p<0.0001)$ and increased AA, DPA and DHA ( $p<0.0001)$; EPA was reduced $(p<0.005)$. The ratio of $\mathrm{AA} / \mathrm{EPA}$ was increased in inflamed mucosa $(\mathrm{p}<0.0001)$ (figure 1).

Conclusion PUFA bioavailability within normal mucosa in UC patients is not significantly different from matched controls; however, inflamed mucosa demonstrates significant differences in the bioavailability of PUFA with a reduction in LA and $\alpha$ LNA and a divergent change in AA and EPA leading to a significant increase in the AA/EPA ratio. These findings
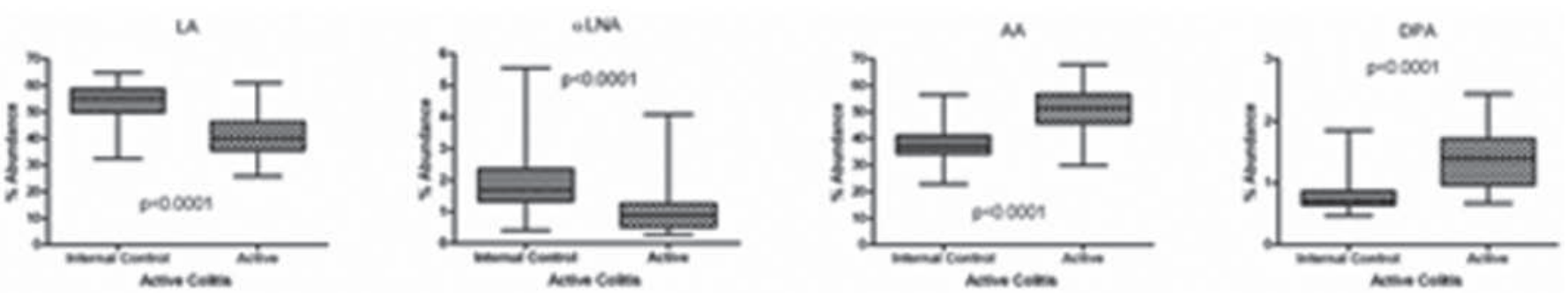

Figure 1 РTH-048

Table 1 PTH-048

\begin{tabular}{|c|c|c|c|}
\hline & Score 4 & Score 2 & Score 1 \\
\hline \multirow[t]{2}{*}{ Disease behaviour } & Panenteric disease & B2 disease OR Perianal disease req 1-2 ops & Single site involvement \\
\hline & OR perianal disease req $\geq 3$ ops $0 R B 3$ disease & & B1 disease \\
\hline \multirow[t]{2}{*}{ Management } & $\begin{array}{l}\text { Steroid dependency } \mathrm{OR} \geq 2 \text { immunomods } \mathrm{OR} 2 \\
\text { resections }\end{array}$ & $\begin{array}{l}\geq 4 \text { steroid courses, none }>4 \text { months } \text { OR } 1 \\
\text { immunomods OR } 1 \text { resection }\end{array}$ & $\begin{array}{l}1-3 \text { steroids courses, each }<4 \text { months; } \\
\text { no immunomods }\end{array}$ \\
\hline & OR biological therapy & & \\
\hline Lowest BMI recorded & $<15$ & $15-18.5$ & \\
\hline Hospitalisations for active disease & $\geq 5$ & $2-4$ & 1 \\
\hline
\end{tabular}


suggest alteration of the fatty acid biosynthetic pathway in inflamed tissue and may suggest that alternative therapeutic strategies are required to modify the eicosanoid profile in UC.

Competing interests None.

Keywords eicosanoids, GC-MS, PUFA, UC. 